Canad. Math. Bull. Vol. 20 (1), 1977

\title{
A THREE-FOLD NON-LATTICE COVERING
}

\author{
W. J. BLUNDON
}

Let $\bar{D}_{k}$ be the density of thinnest $k$-fold covering of the plane by equal circles (of radius 1, say). Let $D_{k}$ be the corresponding density when the centres of the circles are at the points of a lattice $\Lambda$. It is clear that $\bar{D}_{k} \leq D_{k}$.

The problem of finding $\bar{D}_{k}$ for any given $k$ is much more difficult than that of finding $D_{k}$. For single covering Kerschner [3] has proved that $\bar{D}_{1}=D_{1}(=D$, say) $=2 \pi / 3 \sqrt{ } 3$. As for lattice coverings, Blundon [1] has proved $D_{2} / 2 D=1$ and that

$$
D_{3} / 3 D=\frac{1}{288}(15+\sqrt{ } 97) \sqrt{ }(22+10 \sqrt{ } 97)=0.94708 \ldots,
$$

and also that $D_{4} / 4 D=25 \sqrt{ } 3 / 48=0.90210 \ldots$

As for non-lattice coverings, the only known significant result is that of Danzer [2], namely, $\bar{D}_{2} / 2 D \leq 0.97087 \ldots$

The purpose of the present paper is to exhibit a rather simple configuration which gives an estimate for $\bar{D}_{3}$ much stronger than the corresponding lattice result. We prove the following theorem.

TheOREM. $\quad \bar{D}_{3} / 3 D \leq 9(5-2 \sqrt{6})=0.90918 \ldots$, and the corresponding configuration is the union of three translates of an equilateral lattice of side $1+\frac{1}{3} \sqrt{ } 6=1.81649 \ldots$

Proof. Let $\Lambda$ be an equilateral lattice of side $i+\frac{1}{3} \sqrt{6}$ with fundamental triangle $\mathrm{OAB}$ where $\mathrm{O}$ is the origin. Let $\Lambda_{1}, \Lambda_{2}, \Lambda_{3}$ denote the translates of $\Lambda$ in the directions $\mathrm{AB}, \mathrm{BO}, \mathrm{OA}$ respectively by a distance $1 / 3$ in each case. Then $\mathrm{O}_{i} \mathrm{~A}_{i} \mathrm{~B}_{i}$ is a fundamental triangle of the lattice $\Lambda_{i}(i=1,2,3)$.

Let circles of radius 1 be centered at the points of the lattices $\Lambda_{1}, \Lambda_{2}, \Lambda_{3}$. Since the configuration of the fundamental parallelograms of $\Lambda, \Lambda_{1}, \Lambda_{2}, \Lambda_{3}$ as a whole is symmetrical about the line $A B$, it is sufficient to ensure that every point of the triangle $O A B$ is covered by at least three of the circles centred at the vertices of the triangles $\mathrm{O}_{1} \mathrm{~A}_{1} \mathrm{~B}_{1}, \mathrm{O}_{2} \mathrm{~A}_{2} \mathrm{~B}_{2}, \mathrm{O}_{3} \mathrm{~A}_{3} \mathrm{~B}_{3}$.

Construct an equilateral triangle $P_{1} Q_{1} R_{1}$ with points $P_{1}, Q_{1}$ on the line $O_{3} B_{3}$ such that $R_{1}, A_{1}$ are on opposite sides of the line $O_{3} B_{3}$ and such that $\mathrm{O}_{3} \mathrm{Q}_{1}=\mathrm{B}_{3} \mathrm{P}_{1}=1$. This equilateral triangle has side $1-\frac{1}{3} \sqrt{ } 6$. Construct corresponding triangles $P_{2} Q_{2} R_{2}, P_{3} Q_{3} R_{3}$ as in the diagram.

Received by the editors June 17, 1976 and, in revised form, Oct 20, 1976. 


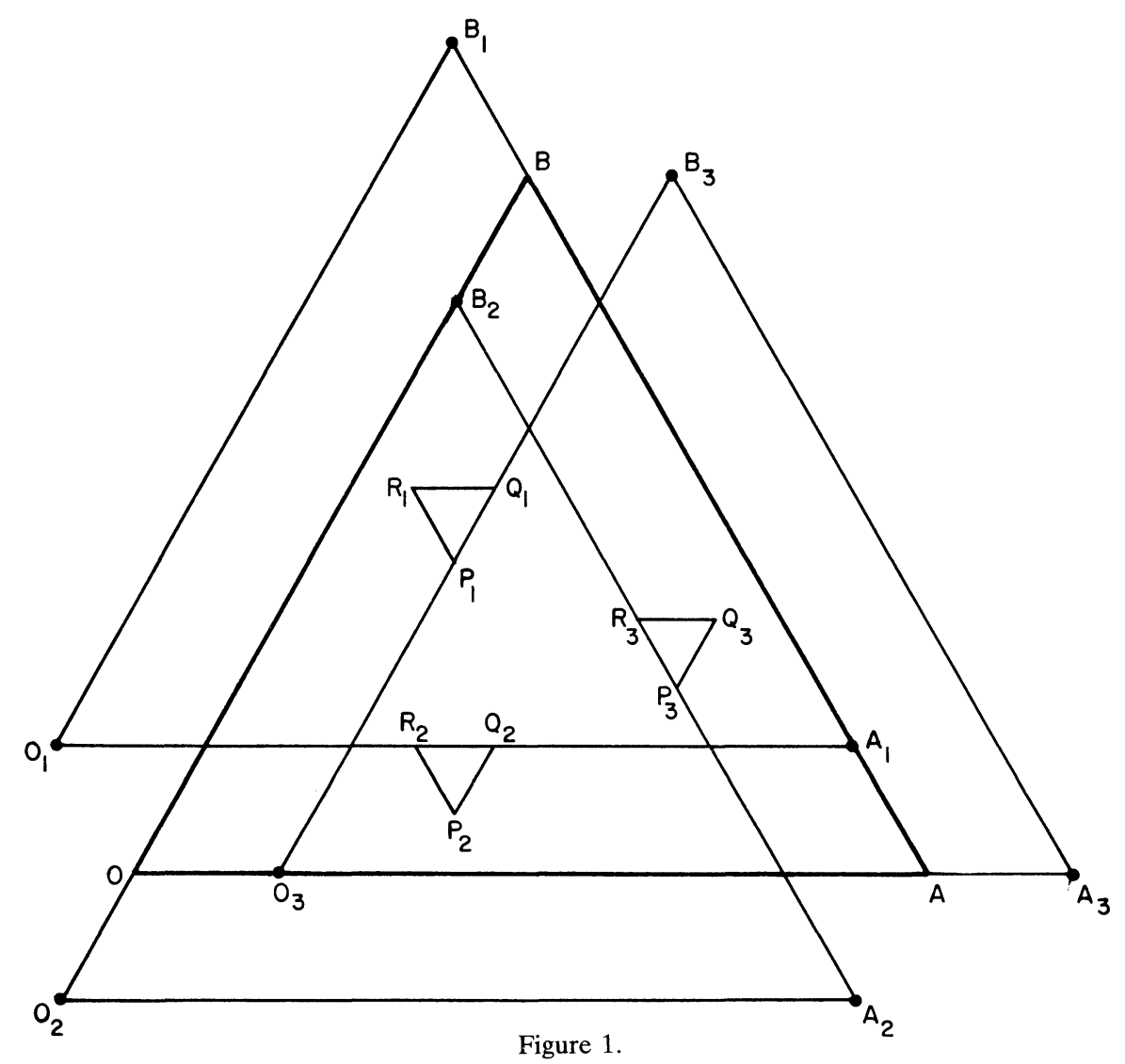

Consider the covering of a triangle $\mathrm{OAB}$ by circles centred at the vertices of the triangle $O_{1} A_{1} B_{1}$. It is easily verified that each of the segments $O_{1} P_{1}, O_{1} R_{1}$, $B_{1} R_{1}, B_{1} Q_{1}, A_{1} Q_{1}, A_{1} P_{1}$ has length 1 . Hence all points of the triangle $O_{1} A_{1} B_{1}$ (with the exception of points in the triangle $P_{1} Q_{1} R_{1}$ ) are covered at least once by the circles at $\mathrm{O}_{1}, \mathrm{~A}_{1}, \mathrm{~B}_{1}$. Also, since the distance from each of $\mathrm{O}_{1}, \mathrm{~A}_{1}$ to the foot of the perpendicular from $\mathrm{P}_{2}$ to $\mathrm{OA}$ is less than 1 , it follows that each of the circle at $\mathrm{O}_{1}$ and the circle at $\mathrm{A}_{1}$ covers all those points of the triangle which are outside the triangle $O_{1} A_{1} B_{1}$. Further, each of these two circles covers the triangle $P_{2} Q_{2} R_{2}$, since $O_{1} Q_{2}=A_{1} R_{2}=1$.

To sum up, the circles of $\Lambda_{1}$ do not cover at all the points of the triangle $P_{1} Q_{1} R_{1}$ but they do cover every other point of the triangle OAB including a double covering of the triangle $\mathrm{P}_{2} \mathrm{Q}_{2} \mathrm{R}_{2}$ and a single covering of the triangle $P_{3} Q_{3} R_{3}$. Repeat the same argument for the circles of $\Lambda_{2}$ and of $\Lambda_{3}$.

Thus each of the triangles $P_{1} Q_{1} R_{1}, P_{2} Q_{2} R_{2}, P_{3} Q_{3} R_{3}$ is covered not at all by one of the lattices, once by a second lattice, and twice by the third lattice. Every other point of the triangle $\mathrm{OAB}$ is covered at least once by each of the three lattices. 
Therefore the given configuration does indeed provide a three-fold covering of the plane. Let $\Delta$ be the determinant of the lattice $\Lambda$. Then $\Delta=\left(\frac{1}{2} \sqrt{3}\right)\left(1+\frac{1}{3} \sqrt{ } 6\right)^{2}$ and $\bar{D}_{3} \leq 3 \pi / \Delta$. Since $D=2 \pi / 3 \sqrt{3}$, it follows that $D_{3} / 3 D \leq 9(5-2 \sqrt{6})=0.90918 \ldots$ This completes the proof of the theorem.

\section{REFERENCES}

1. W. J. Blundon, Multiple coverings of the plane by circles, Mathematika 4 (1957), 7-16.

2. L. Danzer, Drei Beispliele zu Lagerungsproblemen, Arch. Math. 11 (1960), 159-165.

3. R. Kershner, The number of circles covering a set, Amer. J. Math. 61 (1939), 655-671.

Department of Mathematics, Statistics and Computer Scicnce

MEMORIAL UNIVERSITY OF NEWFOUNDLAND

ST. JOHN'S, NEWFOUNDLAND 